\title{
Competition May Increase Social Utility in Bipartite Matching Problem
}

\author{
Yi-Xiu Kong $\mathbb{D}^{1,}{ }^{1,2}$ Guang-Hui Yuan, ${ }^{3}$ Lei Zhou, ${ }^{1}$ Rui-Jie Wu $\mathbb{D}^{1},{ }^{2}$ and Gui-Yuan Shi $\mathbb{D}^{1,2}$ \\ ${ }^{1}$ Faculty of Computer and Software Engineering, Huaiyin Institute of Technology, Huaian 233003, China \\ ${ }^{2}$ Department of Physics, University of Fribourg, Fribourg 1700, Switzerland \\ ${ }^{3}$ Fintech Research Institute, Shanghai University of Finance and Economics, Shanghai 200433, China
}

Correspondence should be addressed to Gui-Yuan Shi; guiyuan.shi@unifr.ch

Received 12 June 2018; Revised 19 September 2018; Accepted 23 October 2018; Published 1 November 2018

Guest Editor: Claudio Tessone

Copyright (C) 2018 Yi-Xiu Kong et al. This is an open access article distributed under the Creative Commons Attribution License, which permits unrestricted use, distribution, and reproduction in any medium, provided the original work is properly cited.

\begin{abstract}
Bipartite matching problem is to study two disjoint groups of agents who need to be matched pairwise. It can be applied to many real-world scenarios and explain many social phenomena. In this article, we study the effect of competition on bipartite matching problem by introducing conformity into the preference structure. The results show that a certain amount of competition can improve the overall utility of society and also eliminate the giant shift of social utility when matching unequal numbers of men and women.
\end{abstract}

\section{Introduction}

Bipartite matching problem is to study how the two disjoint groups of agents can be matched pairwise for their personal preferences, such as the matching between men and women, students and colleges, workers and jobs, consumers and products [1-3], and many other scenarios [4-7]. For convenience we use the paradigm of marriage problem, where $\mathrm{N}$ men and $\mathrm{N}$ women need to be matched. In this problem, each participant is selfish, everyone tries to optimize their own choices, and the competition is inevitable. A key question is how to find a stable solution in which there is no such a pair of man and woman who prefer themselves more than the assigned partner [8]. The number of stable solutions is very large [9]; the most famous one among them is obtained by the Gale-Shapley algorithm, which was awarded the Nobel Prize in Economics in 2012.

In 1962, Gale and Shapley proved that for the same number of men and women, a stable solution can always be obtained through Gale-Shapley algorithm [1]. In this algorithm, every agent has a preference list, which is a ranking list of all members from the opposite sex. Men act as suitors and send proposals to women according to their preference lists. When a woman has multiple candidate partners, she always retains her favorite one. The algorithm will continue until all agents find their spouses. This matching result can easily prove to be stable, because the only way for men to improve their current situation is to send proposals to women who have already rejected them, but the spouse of these women must be in front of this man in the women's lists. Besides, it also proved to be the men-optimal solution among all stable solutions.

Statistical physicists also find areas of interest in the bipartite matching problem because the model is very similar to a system in which two different particles interact $[10,11]$. We assume that the utility of an agent is corresponding to the ranking of her/his spouse in her/his preference list [12]. It can be generally regarded as a cost function, or 'energy' in physics terminology. In the following text, the term 'energy' of an agent is used to express the utility of an agent. If a person just happens to match the person at the top of her/his preference list as a spouse, then she/he will be the happiest and have an 'energy' of 1 . In the worst case, one had to choose the person at the bottom of the list and the 'energy' would be $N$. In most of the previous researches $[9,12,13]$, for simplicity, the preference lists are always established randomly and independently. 
Physicists are constantly interested in finding a solution with the lowest 'energy', which is called the ground state [1316]. This is equivalent to the commonly used term 'minimal cost solution'. The replica method in spin glass is used to compute the minimal cost solution in the bipartite matching problem [14], and the result gives that the average 'energy' of each individual in the minimal cost solution is $0.808 \sqrt{N}$ [12]. However, $24.2 \%$ of men can find one or more women [13], so that both of them prefer each other to their current spouses. Therefore, this minimal cost solution is a very unstable matching. Moreover, the Mean Field Theory is utilized to calculate the average 'energy' of the stable solution obtained by Gale-Shapley algorithm [12]. In this case, the average 'energy' of men is $\log (N)$, and the average 'energy' of women is $N / \log (N)$. Further, with the aid of the 'energy' distribution function, it is proved that for all stable solutions, there exists a relationship $\epsilon_{m} * \epsilon_{w}=N$. The average 'energy' is $\epsilon_{g}=\left(\epsilon_{m}+\right.$ $\left.\epsilon_{w}\right) / 2=0.5\left(\epsilon_{m}+N / \epsilon_{m}\right)$. It is easy to see that the global optimal stable solution corresponds to the same 'energy' $\sqrt{N}$ for both men and women, which is a little worse than the global optimal solution. In addition, it is worthwhile to mention that the men-optimal solution has the highest total 'energy' among all stable solutions. After that, various issues were investigated, such as the matching problem under partial choice $[17,18]$, which means the lists of men only contain a fraction of all women, or people have a spatial distribution and tend to match people who are geometrically closer [19], etc.

One of the interesting questions is, if people's preference lists are not strictly random, i.e., if we induce a conformity term into the preference structure, i.e., the agents have similar preferences, which makes some of the agents become more popular than others for all agents, how will the popularity of agents affect the matching results? Later we will show that the weight of popularity in the preference structure is directly related to the competition intensity. In previous studies [19], numerical simulation showed that the exerting of competition will lead to higher 'energy' and lower utility. Here we thoroughly study the impact of competition on bipartite matching, and instead of intuitive result that competition may reduce the utility of the society, our result shows that a certain amount of competition can increase the social utility. On the other hand, a recent research [20] shows that random bipartite matching is very sensitive to the symmetry of the sizes of the two sides; even reducing the number of women by only one will lead to a dramatic change of the 'energy' in the matching result. However, this dramatic change in social utility is rarely observed in real life, even though the numbers of matching parties in reality are often different. Our research shows that introducing competition into the preference list can effectively decrease the symmetry sensitivity of the matching result and explain the absence of the dramatic change in daily observation.

\section{Method}

The Gale-Shapley algorithm [1] is described below: suppose we have two disjoint sets of $\mathrm{N}$ men and $\mathrm{N}$ women who need to be matched pairwise. Each person has her/his preference list which stores the ranking of all members from the opposite sex. At the beginning, everyone is unengaged. In each step, each unengaged man issues a proposal to his favorite woman among those he has not proposed to. The courted woman will choose her favorite from all the suitors and her provisional partner. The process iteratively runs until everyone is matched and it is easy to realize that a final matching will be achieved eventually.

\section{Results and Discussions}

3.1. Matching between $N$ Men and N Women. Let us firstly consider the matching problem when the two groups have equal size. For simplicity, it is usually assumed that everyone's preference list is completely random. Considering the process of Gale-Shapley algorithm, men make proposals to women. If the courted woman is unengaged, then the total number of matched pairs will increase by one. If this woman is engaged, no matter the suitor or her current partner is retained, the number of partners will not change.

It is proved that on average [12], a total number of $N(\log (N)+0.522)$ proposals need to be sent to make everyone engaged. Every proposal leads to a man's 'energy' increasing by one; the average 'energy' of men is the same as the average number of proposals that he needs to make; i.e., the average 'energy' of men is $\log (N)+0.522$. On the other hand, for women, each of them receives on average $\log (N)+$ 0.522 proposals. Each time they receive a proposal, they can make a choice and decide the man whom they prefer. Obviously, the more proposals they receive, the lower 'energy' women have. Since each suitor is randomly distributed in the preference list, the optimal one among many choices is equivalent to the first order statistic of multiple random sampling from uniform distribution. It is easy to obtain the woman's average 'energy' as $N /(\log (N)+0.522+1)$. When $N$ is large, the average male 'energy' is approximately equal to $\log (N)$, and the average female 'energy' is approximately equal to $N / \log (N)$. This conclusion is consistent with the results of the 'energy' distribution function method used in previous study [12].

However, in reality the preference lists are seldom purely random. Some intrinsic properties, such as beauty, intelligence, and wealth, will affect the ranking order of the preference lists and then a certain level of conformity can be observed in the structure of their preference lists. People with those widely accepted attributes are easier to rank in front of the preference lists of all agents. Let us consider the extreme case, when the preferences of all agents are strictly based on popularity term; then everyone should have an identical preference list. At the first step, all men will make proposals to the woman they all prefer. The man ranked at the top of women's preference list will be accepted and the other men are refused. After that, the remaining men make proposals to the woman who is their second favorite, the man ranked second in the women's preference lists will be accepted and the others will be rejected. The process continues, it is easy to know that the men's 'energy' from low to high are $1,2,3 \ldots N$ 


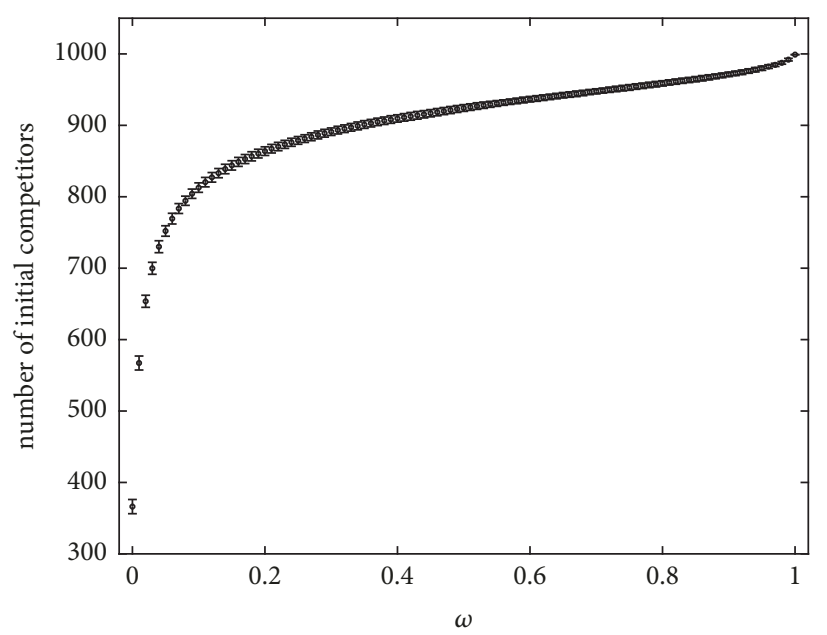

FIGURE 1: The number of initial competitors versus the weight of popularity, $N=1000$; the result is averaged over 100 realizations.

and the average 'energy' $(N+1) / 2$. The average 'energy' of women is the same.

We define the men who have been rejected after the first round of proposing as the initial competitors. Thus, the total number of initial competitors can be regarded as a measure of the competition intensity of the matching. In the extreme case above (preference lists are completely the same), following the Gale-Shapley algorithm, all men will propose to the same woman so that the number of initial competitors is $N-1$. Now consider the other extreme case when the preference lists are purely random; theoretically the probability that a woman does not receive any proposal is $(1-1 / N)^{N}$; when $N$ is large, this probability is close to $1 / e$. Since the number of men who have been rejected is equal to the number of women who have not received any proposals, the number of initial competitors is $N / e \simeq 0.368 \mathrm{~N}$.

In general, we propose a model to characterize the realistic situation that some of the agents are ranked high in the preference lists of all the opposite sex agents. In this model the scores of each agent rating the agents of the opposite sex consist of two parts: the popularity term that reveals the conformity of the preference lists and a random term that brings diversification of the personal preferences.

We assume that woman $i$ rates man $\alpha$ with a score $S_{\alpha, i}$.

$$
S_{\alpha, i}=\omega \times F_{\alpha}+(1-\omega) \times N_{\alpha, i}
$$

Here $F_{\alpha}$ is popularity of man $\alpha$ and $N_{\alpha, i}$ is a random term. For simplicity, we assume that $F$ and $N$ are uniformly distributed on $[0,1]$ and that weight of popularity $\omega$ is universal for all men and women. One can choose other distributions for the popularity, but it does not change the main conclusion. For any intermediate situations the number of initial competitors lies in $[0.368 \mathrm{~N}, \mathrm{~N}]$ because of the monotonous relationship of $S_{\alpha, i}$ and the weight $\omega$ by definition.

Below we present a numerical simulation result (Figure 1) which shows how the number of initial competitors changes with the weight of popularity in our model. The simulation is done on a system containing $\mathrm{N}=1000$ agents on each side, and the result is averaged over 100 realizations. As shown in Figure 1, the number of initial competitors gets increasingly higher as the weight of conformity grows.

The preference list of woman $i$ is generated according to the order of her ratings of all men. Similarly, we can obtain the preference list of everyone. With these preference lists, implementing Gale-Shapley algorithm, we will obtain the final stable matching.

Now we start to analyze this matching result. As shown in Figure 2(a), the average 'energy' of men increases monotonically with the weight of popularity, $\omega$. This is because when the weight of popularity increases, more proposals are required for everyone to be engaged. However, a slight competition will significantly reduce the women's 'energy?. This increase of women's average 'energy' grows with $N$ (Figure 2(b)), and the competition intensity required for the women to reach optimal 'energy' decreases as the population grows (Figure 2(c)). As competition further intensifies, the women's 'energy' will also increase. On the one hand, due to the increase of the number of proposals, the number of choices of woman increases. It can be known from the nature of the order statistics that the minimum number of multiple random sampling decreases as the number of sampling increases. The increase in competition between men is beneficial to the women. On the other hand, the increase in the weight of popularity also leads women to tend to favor the men with high popularity and thus increases the intensity of competition among women. Many women have to be matched with the men positioned further down on their preference lists. This will lead to an increase in the average 'energy' of women and all people.

Due to the increase of $\omega$ in the beginning, the reduction of women's 'energy' is more significant than the increase of men's 'energy'. As shown in Figure 3(a), there exists a $\omega_{g}^{*}$ that can make average 'energy' of all people reaches the optimal value. In other words, it is a certain degree of social popularity that increases the total social utility.

In order to study the relationship between a person's intrinsic popularity and her/his level of utility, we cluster 

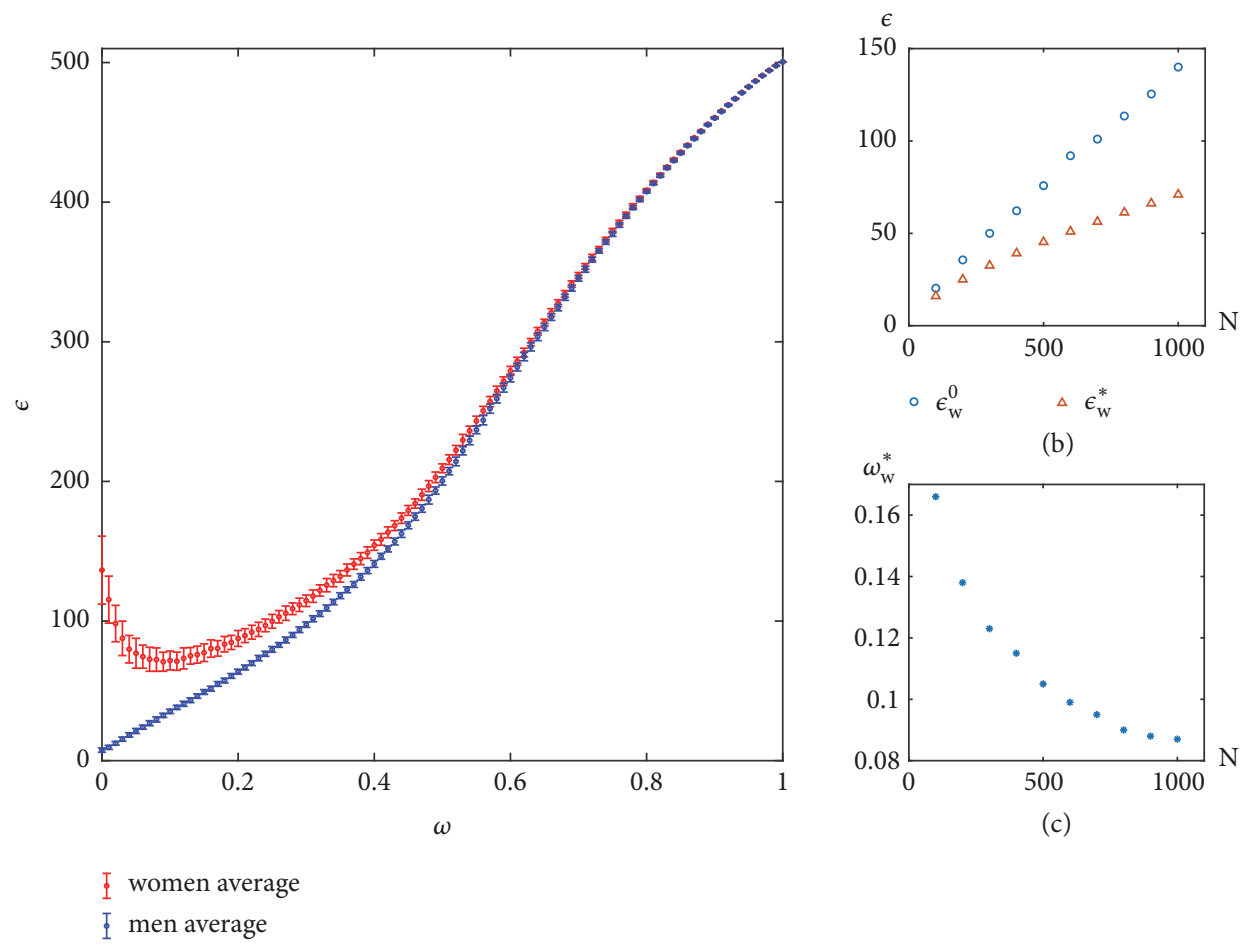

(a)

Figure 2: The numerical simulation of average 'energy' of men and women, $N=1000$; the result is averaged over 100 realizations. (a) The average 'energy' of men and women, as a function of $\omega$; (b) for $N=100,200,300, \ldots, 1000$, the average 'energy' of women at $\omega=0$ is denoted by $\epsilon_{w}^{0}$ and at $\omega=\omega_{w}^{*}$ is denoted by $\epsilon_{w}^{*}$. Here $\omega_{w}^{*}$ is the women-optimal value of conformity weight; (c) the optimal weight $\omega_{w}^{*}$ versus $N$.
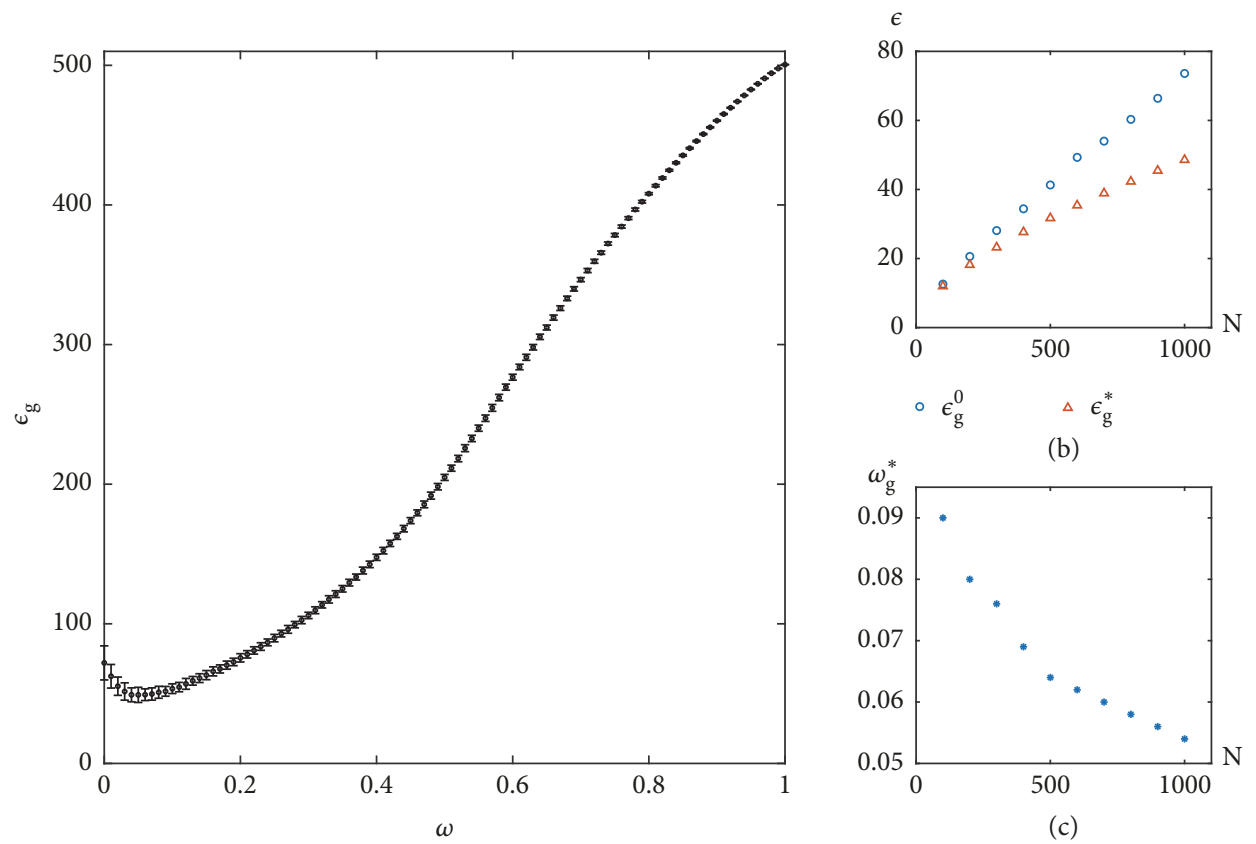

(a)

FIgURE 3: The numerical simulation of average 'energy' of all agents, $N=1000$; the result is averaged over 100 realizations. (a) The average 'energy' of all agents, as a function of $\omega$; (b) for $N=100,200,300, \ldots, 1000$, the average 'energy' at $\omega=0$ is denoted by $\epsilon_{g}^{0}$ and at the global optimal popularity weight $\omega_{g}^{*}$ is denoted by $\epsilon_{g}^{*}$; (c) the optimal weight $\omega_{g}^{*}$ versus $N$. 

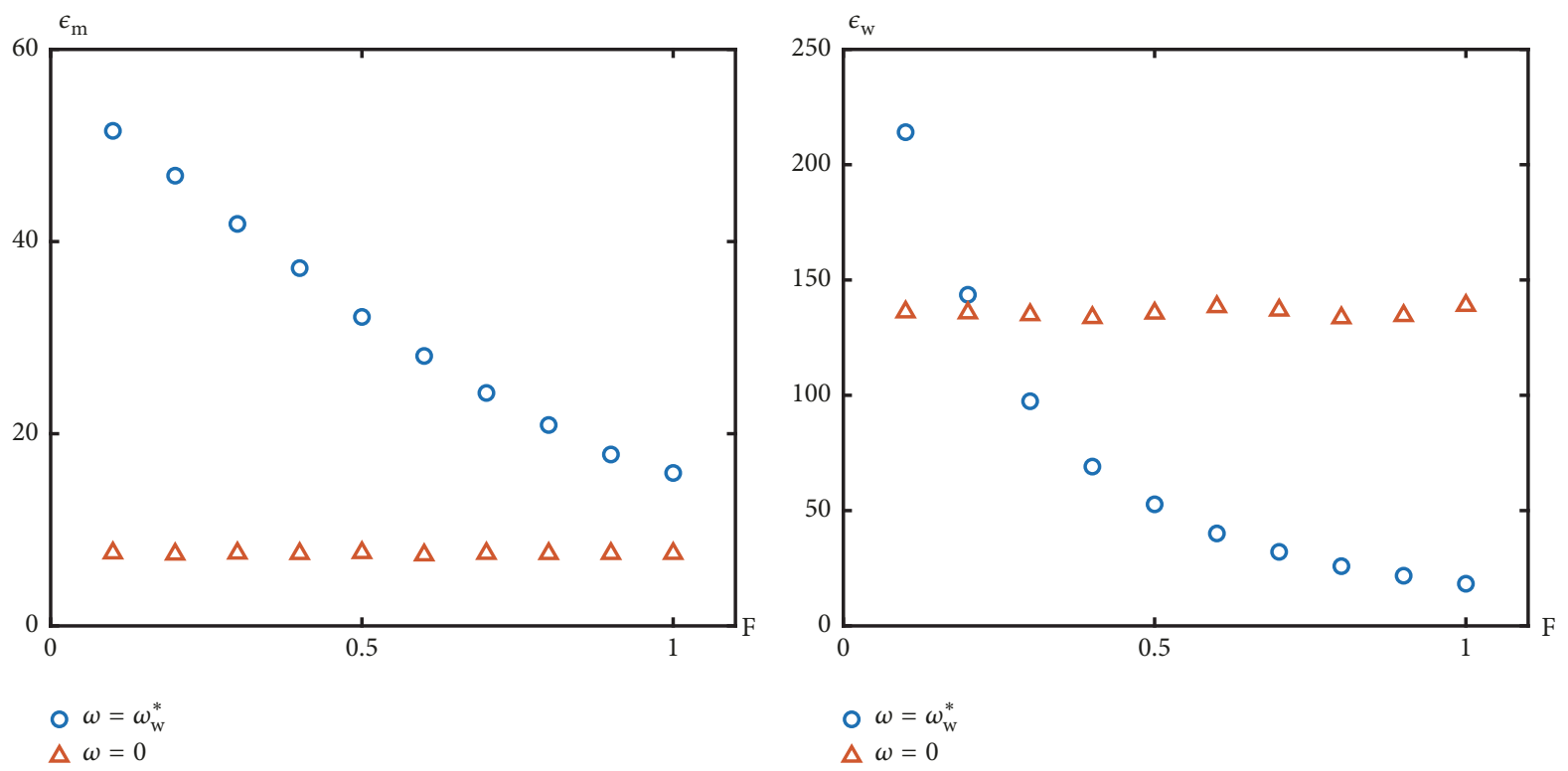

(a)

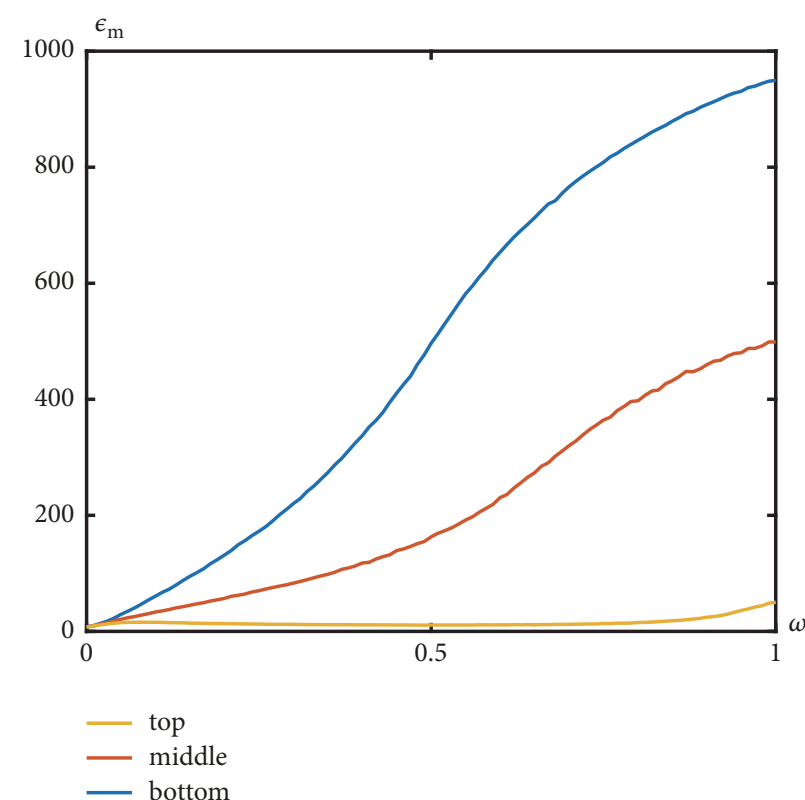

(c) (b)

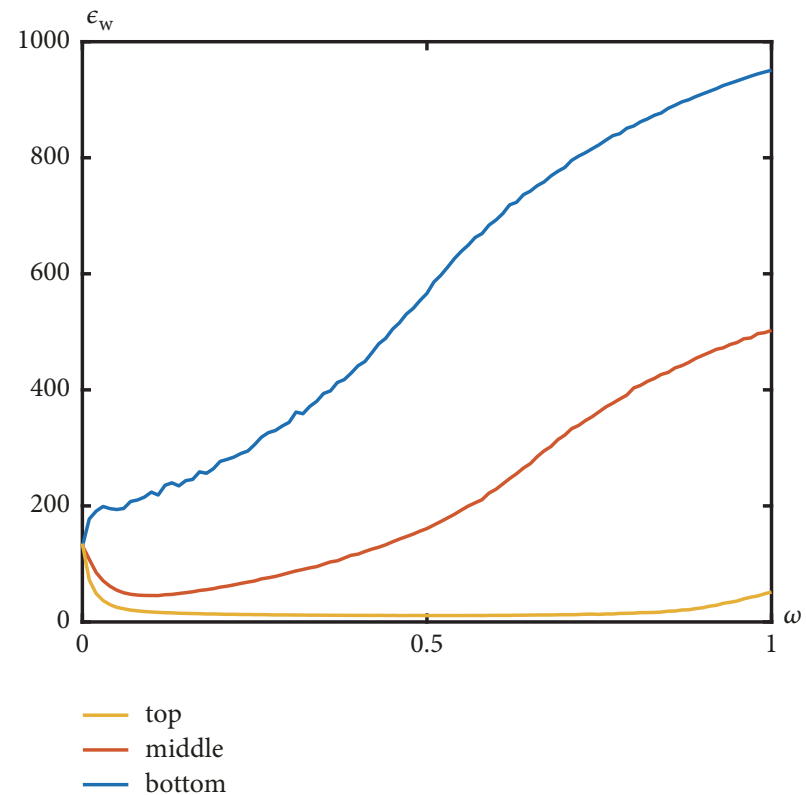

(d)

Figure 4: The average 'energy' of different popularity groups; the horizontal axis shows the different values of popularity. $N=1000$; the result is averaged over 100 realizations. (a) The average 'energy' of men at $\omega=0$ and $\omega=\omega_{w}^{*}$; (b) the average 'energy' of women at $\omega=0$ and $\omega=\omega_{w}^{*}$; (c) the average 'energy' of three representative popularity groups of men, bottom $(0,0.1)$, middle $(0.45,0.55)$, and top $(0.9,1)$, versus $\omega$; (d) the average 'energy' of three representative popularity groups of women, bottom $(0,0.1)$, middle $(0.45,0.55)$, and top $(0.9,1)$, versus $\omega$. Note that when $\omega=0$ (triangles) the average 'energy' of men or women stays the same as the value shown in Figure 2(a); the small deviations are the random fluctuations which are irrelevant to the popularity.

the 'energy' of men and women to 10 groups according to the range of popularity $[0,0.1),[0.1,0.2), \ldots,[0.9,1]$ (data binning) and compare their utility in two cases: $\omega=0$ and $\omega=\omega_{w}^{*}$ (women's optimal). As shown by Figure 4(a), the competition causes 'energy' of men to rise in all popularity groups. As shown in Figure 4(b), at the women's optimal weight of popularity, the 'energy' of women in lowest $20 \%$ conformity groups increases, and the 'energy' of the remaining $80 \%$ women decreases. In general, women will have lower 'energy' with a certain level of competition, as we have known above.

In addition, we take three representative agent groups and label them as bottom (popularity ranges $[0,0.1]$ ), middle (conformity ranges $[0.45,0.55]$ ), and top (popularity ranges $[0.9,1])$, respectively. With the increase of competition, i.e., the increase of $\omega$, the 'energy' of the three groups of men 

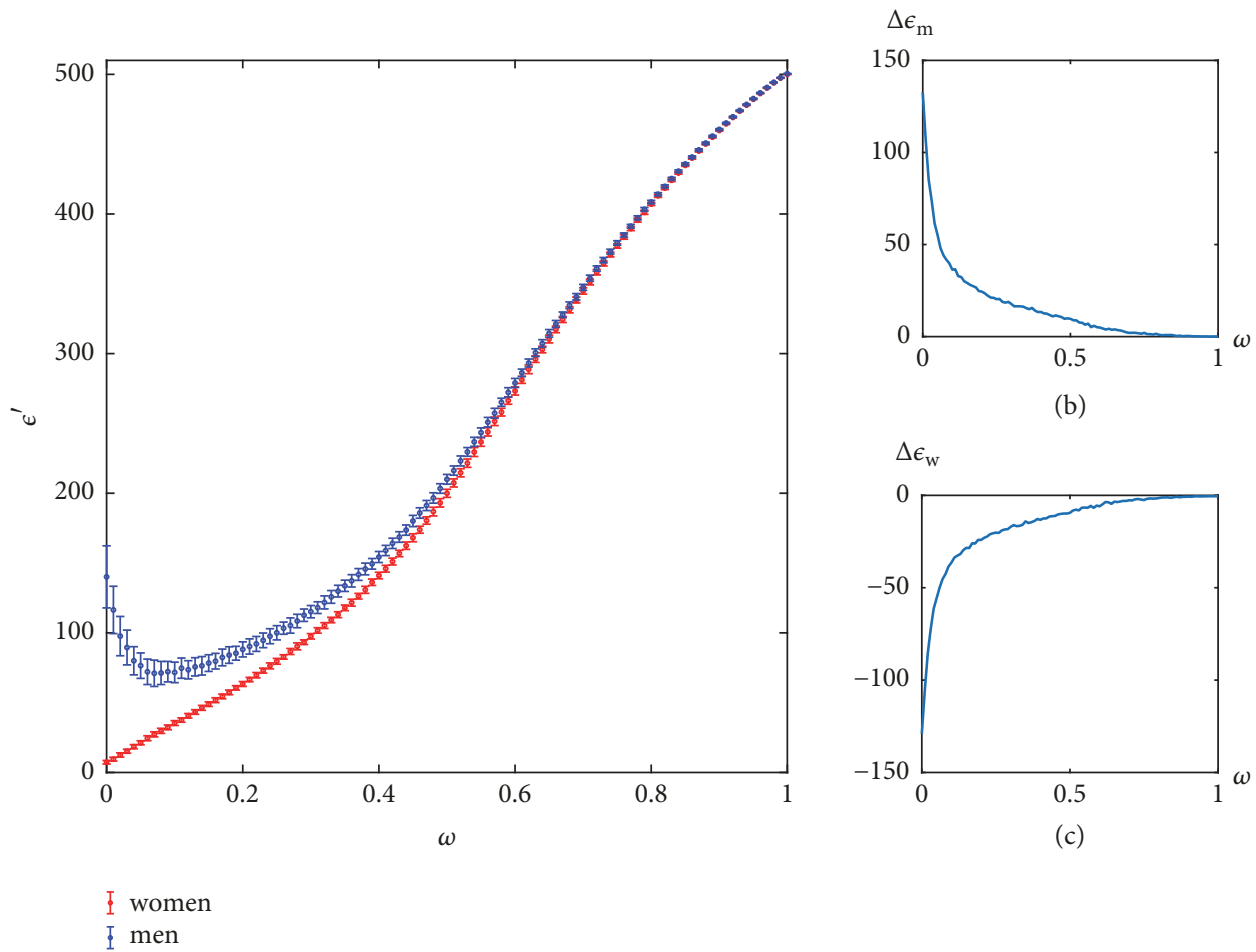

(b)

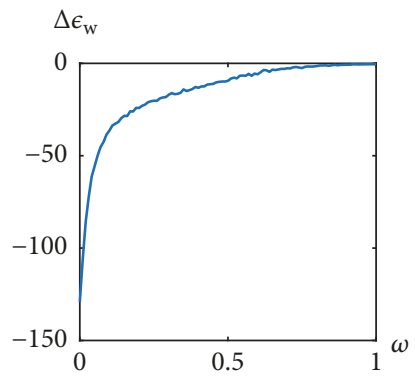

(c)

(a)

FIgURE 5: The matching between 1000 men and 999 women, as a function of $\omega$. The result is averaged over 100 realizations. (a) The average 'energy' of men and women; (b) $\Delta \epsilon_{m}$ versus $\omega$; (c) $\Delta \epsilon_{w}$ versus $\omega$.

will increase, while the top women will have lower 'energy' and the bottom women will have higher 'energy' (as shown in Figures 4(c) and 4(d)). This change is very sensitive to $\omega$; even if $\omega$ is very small (0.01), the 'energy' difference between the top group and bottom group has an obvious gap. For the middle group women, a slight competition improves their utility, but as the competition intensifies, their 'energy' becomes even higher.

3.2. Matching between $N$ Men and $N-1$ Women. For a long time, the G-S algorithm has been considered to produce a men-optimal stable matching. In particular, for a completely random preference list, that is, when $\omega=$ 0 , the average 'energy' of men is $\log (N)+0.522$, which is far less than the average 'energy' of women, which is $N /[\log (N)+0.522]$, so the active side takes a huge advantage in the matching. However, a recent research [20] shows that in a random bipartite matching, if one woman is removed from the matching, the average 'energy' of men will become $N / \log (N)$, and the average 'energy' of women will become $\log (N)$. The 'energies' of the positive and passive sides are completely reversed.

In our daily life, the sizes of matching parties are rarely equal. Imagine removing one agent of passive party in the bipartite matching, for example, reducing a woman in the marriage problem, downsizing one job position in the labor market, or cutting one offer in the college admission; under the assumption of random bipartite matching, the pairing result will change drastically with the smallest change of the size of passive side. In particular in the marriage problem, due to the natural inequality of birth rates of different sex as well as some other cultural or political impact, the sex ratio (boys versus girls) is larger than 1. It makes the matching of unequal numbers of men and women have even more practical meaning. However, in reality, one of the possible reasons for the phenomenon that such a large difference in social utility is rarely seen, is that the low-popularity agents will be quickly eliminated and other people's pairing results are almost unaffected.

We analyze the matching results in the case of 1000 men and 999 women with different $\omega$. As shown in Figure 5(a), the curves are extremely similar to that of Figure 2(a), despite the fact that the gender has reversed. The women become the dominant side of the matching. We compare this asymmetric case with the symmetric case above and examine the changes of the average 'energy' of men and women, $\Delta \epsilon_{m}=\epsilon_{m}^{\prime}-\epsilon_{m}$ and $\Delta \epsilon_{w}=\epsilon_{m}^{\prime}-\epsilon_{w}$. The results are shown in Figures 5(b) and 5(c), respectively. It is found that this difference sharply decreases with the increase of $\omega$, which explains why the slight change in the number of people in reality does not obviously affect overall utility society.

\section{Conclusion}

In this article we introduce competition in bipartite matching problem by bringing the conformity in the preference lists 
of agents. While in the traditional Gale-Shapley model, the preference lists of agents are randomly generated, the active agents can easily acquire the results they want and stop sending proposals so that the agents from the passive side are left with little freedom of choice. We show that if a certain amount of competition is introduced, the active agents have to make more efforts and send more proposals, which will slightly decrease their utility. However, at the same time, the utility of the passive side will be obviously increased, and society as a whole will have a higher total utility than the original matching result. To summarize, a certain amount of competition increases the total utility of the society. This not only is true for the bipartite matching problem, but also enlightens our understanding of many other social phenomena. Besides, when matching two parties of different sizes, the utility of the two parties can be dramatically changed compared to the symmetric case. The introduction of conformity in the preference lists can also eliminate this potential significant utility change, which provides a possible explanation why this significant utility change is rarely seen in human society.

\section{Data Availability}

No data were used to support this study.

\section{Conflicts of Interest}

The authors declare no conflicts of interest.

\section{Acknowledgments}

We would like to thank Prof. Yi-Cheng Zhang and Wen-Yao Zhang for helpful discussions. This research is supported in part by the Chinese National Natural Science Foundation under grant No. 61602202 and Natural Science Foundation of Jiangsu Province under grants No. BK20160428, BK20161302. Gui-Yuan Shia and Yi-Xiu Kong acknowledge the support from China Scholarship Council (CSC).

\section{References}

[1] D. Gale and L. S. Shapley, "College admissions and the stability of marriage," The American Mathematical Monthly, vol. 69, no. 1, pp. 9-15, 1962.

[2] A. E. Roth, "The Evolution of the Labor Market for Medical Interns and Residents: A Case Study in Game Theory," Journal of Political Economy, vol. 92, no. 6, pp. 991-1016, 1984.

[3] A. E. Roth and M. A. Sotomayor, Two-sided matching, vol. 18 of Econometric Society Monographs, Cambridge University Press, Cambridge, 1990.

[4] B. M. Maggs and R. K. Sitaraman, "Algorithmic nuggets in content delivery," Computer Communication Review, vol. 45, no. 3, pp. 52-66, 2015.

[5] D. Lebedev, F. Mathieu, and L. Viennot, "On using matching theory to understand P2P network design," in In INOC 2007, International Network Optimization Conference, 2007.
[6] M. Hasan and E. Hossain, "Distributed resource allocation in 5G cellular networks," in Towards 5G: Applications, requirements and candidate technologies, pp. 245-269, Wiley, 2015.

[7] G. J. Hitsch, A. Hortaçsu, and D. Ariely, "Matching and sorting in online dating," American Economic Review, vol. 100, no. 1, pp. 130-163, 2010.

[8] A. E. Roth, "The economics of matching: stability and incentives," Mathematics of Operations Research, vol. 7, no. 4, pp. 617628, 1982.

[9] M. Dzierzawa and M.-J. Oméro, "Statistics of stable marriages," Physica A: Statistical Mechanics and its Applications, vol. 287, no. 1-2, pp. 321-333, 2000.

[10] Y. Zhang, "The Information Economy," in Non-Equilibrium Social Science and Policy, Understanding Complex Systems, pp. 149-158, Springer International Publishing, Cham, 2017.

[11] A. Chakraborti, D. Challet, A. Chatterjee, M. Marsili, Y.-C. Zhang, and B. . Chakrabarti, "Statistical mechanics of competitive resource allocation using agent-based models," Physics Reports, vol. 552, pp. 1-25, 2015.

[12] M.-J. Oméro, M. Dzierzawa, M. Marsili, and Y.-C. Zhang, "Scaling behavior in the stable marriage problem," Journal de Physique II, vol. 7, no. 12, pp. 1723-1732, 1997.

[13] G.-Y. Shi, Y.-X. Kong, H. Liao, and Y.-C. Zhang, "Analysis of ground state in random bipartite matching," Physica A: Statistical Mechanics and its Applications, vol. 444, pp. 397-402, 2016.

[14] M. Mézard and G. Parisi, "Replicas and optimization,” Journal de Physique Lettres, vol. 46, no. 17, pp. 771-778, 1985.

[15] M. Mézard and G. Parisi, "The Euclidean matching problem," Le Journal de Physique, vol. 49, no. 12, pp. 2019-2025, 1988.

[16] V. S. Dotsenko, "Exact solution of the random bipartite matching model," Journal of Physics A: Mathematical and General, vol. 33, no. 10, pp. 2015-2030, 2000.

[17] Y.-C. Zhang, "Happier world with more information," Physica A: Statistical Mechanics and its Applications, vol. 299, no. 1-2, pp. 104-120, 2001.

[18] P. Laureti and Y.-C. Zhang, "Matching games with partial information," Physica A: Statistical Mechanics and its Applications, vol. 324, no. 1-2, pp. 49-65, 2003.

[19] G. Caldarelli and A. Capocci, "Beauty and distance in the stable marriage problem," Physica A: Statistical Mechanics and its Applications, vol. 300, no. 1-2, pp. 325-331, 2001.

[20] G. Shi, Y. Kong, B. Chen, G. Yuan, and R. Wu, "Instability in Stable Marriage Problem: Matching Unequally Numbered Men and Women," Complexity, vol. 2018, 5 pages, 2018. 


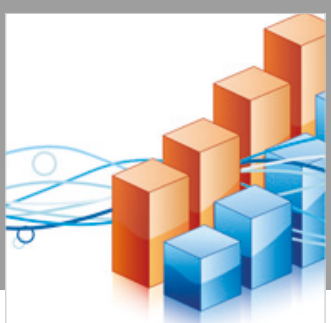

Advances in

Operations Research

\section{-n-m}
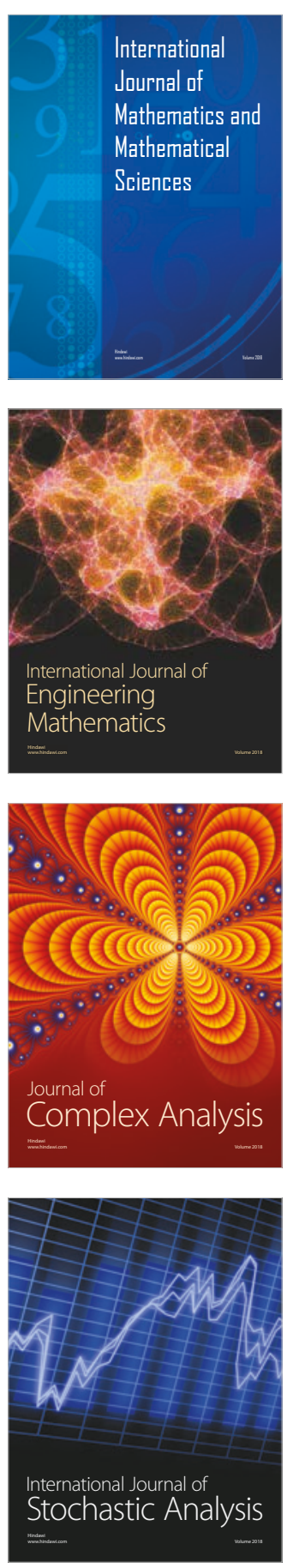
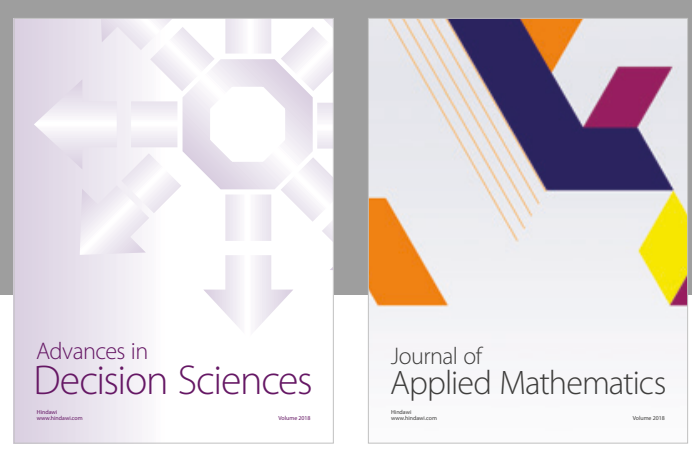

Journal of

Applied Mathematics
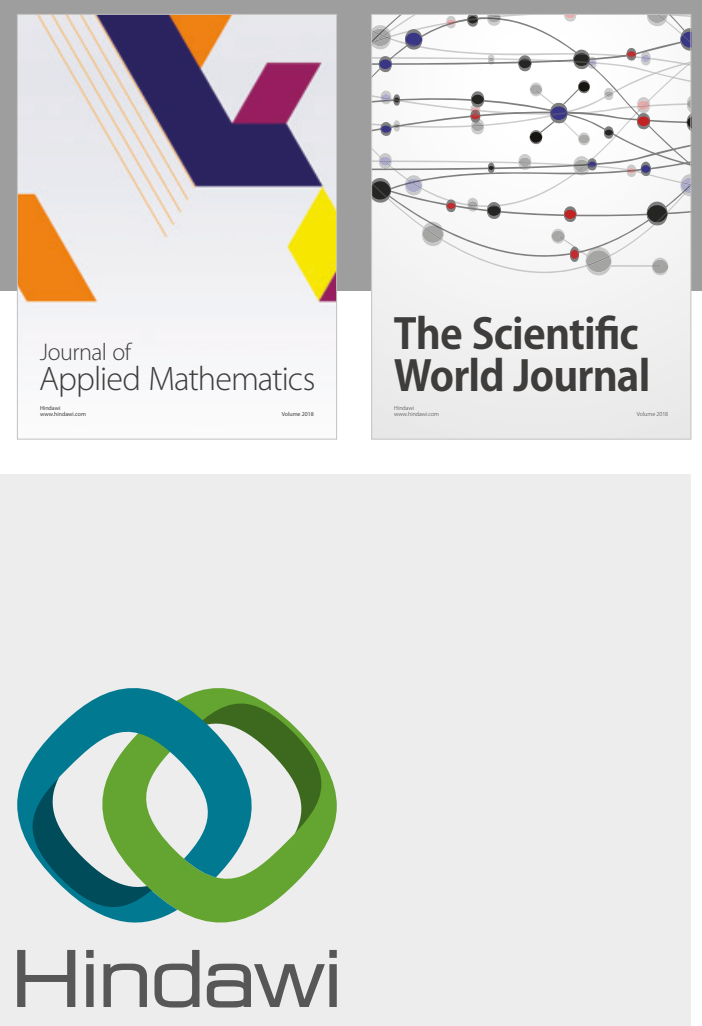

Submit your manuscripts at

www.hindawi.com

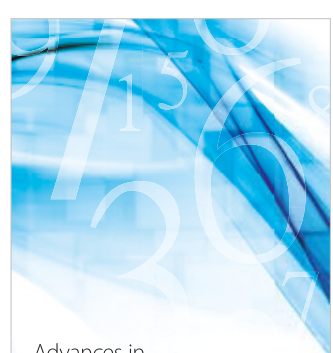

Advances in
Numerical Analysis
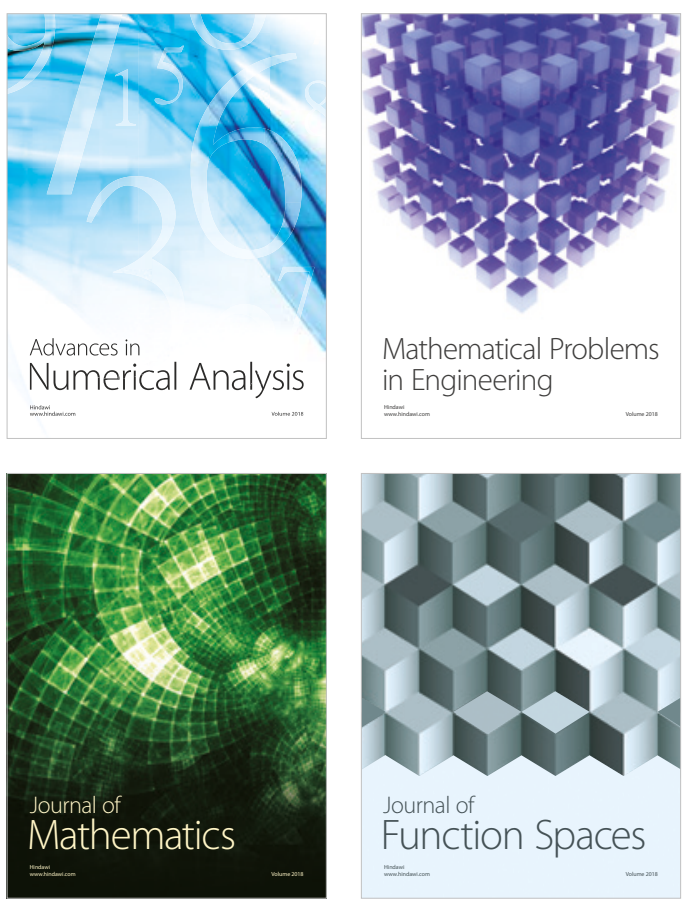

Mathematical Problems in Engineering

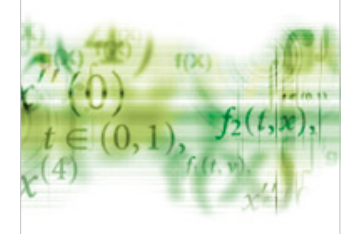

International Journal of

Differential Equations

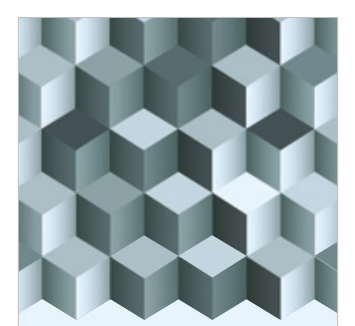

Journal of

Function Spaces
The Scientific

World Journal

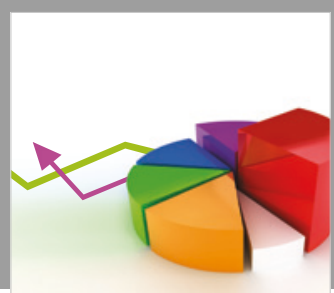

Journal of

Probability and Statistics
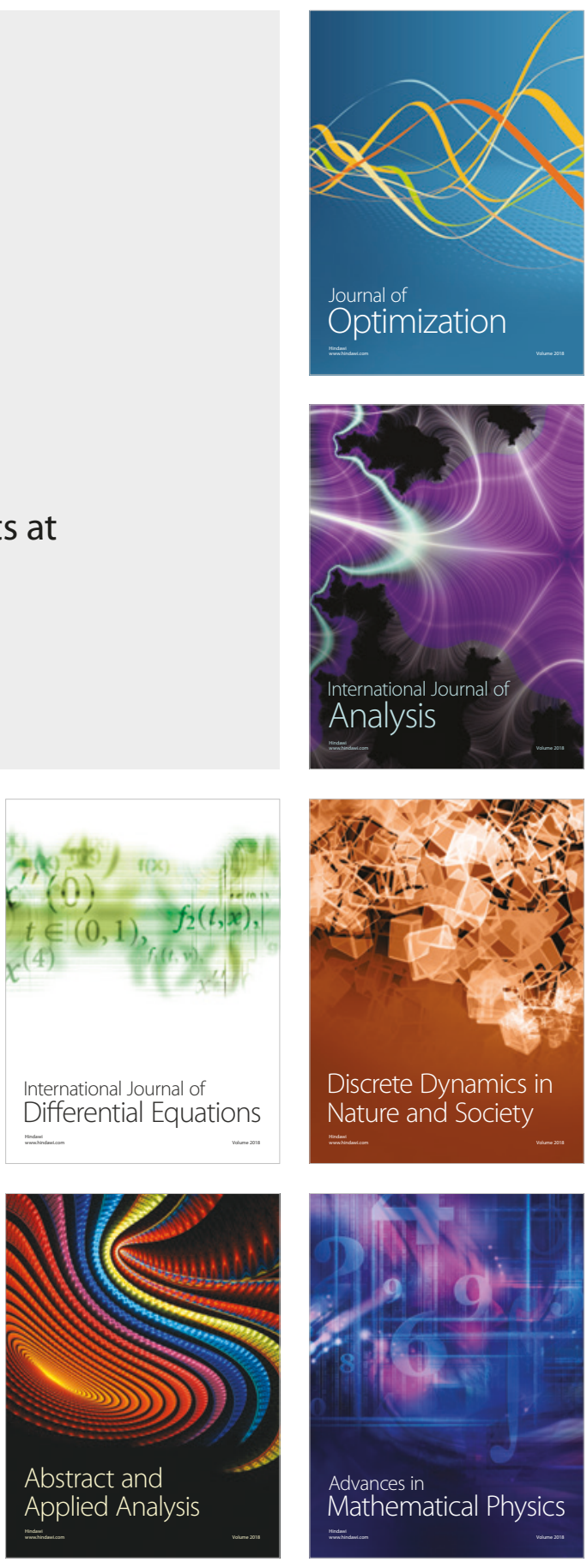